\title{
Les biocarburants en Argentine : facteurs et enjeux de la production de biodiesel de soja
}

\author{
Martine GUIBERT ${ }^{1}$ \\ Silvina Cecilia CARRIZO \\ 1 Université de Toulouse - Le Mirail, \\ Département de Géographie, \\ Equipe de recherche "Dynamiques \\ rurales", 31058 Toulouse cedex 09 \\ < guibert@univ-tlse2.fr> \\ 2 Conicet-CEUR (Centro de Estudios \\ Urbanos y Regionales) et UNNOBA \\ (Universidad Nacional del Noroeste \\ de la Provincia de Buenos Aires), \\ Argentine
}

La fabrication de biocarburants se trouve au carrefour entre agrégation de valeur à des matières premières agricoles et obtention de biens énergétiques renouvelables. Dotée d'une production d'huile brute de soja conséquente et destinée à l'exportation, l'Argentine fournit ainsi, depuis 2007, la demande mondiale croissante en biodiesel de soja ${ }^{1}$. Elle a également le souci d'améliorer l'approvisionnement de son marché national, les besoins énergétiques augmentant et l'extraction d'hydrocarbures devenant insuffisante. De fait, la consommation de biocarburants est obligatoire depuis 2010, et l'objectif est d'incorporer, en 2012, $10 \%$ de biocarburants aux carburants, ce qui permettra de réduire les importations de gaz et de gazole. Ce marché national est capté prioritairement par des acteurs de petite ou moyenne taille et détenteurs de quotas

\footnotetext{
${ }^{1}$ Elle réactive également sa production, modeste par rapport au Brésil, d'éthanol de canne et projette le développement de celle d'éthanol de maïs et de biodiesel de canola (colza), ou encore celles de biocarburants à base $d^{\prime}$ algues ou de déchets organiques.
}

\begin{abstract}
Biofuel production in Argentina: factors and challenges of soybean biodiesel

In 2006, Argentina has sanctioned a national law to regulate biofuel production and consumption. Since 2007, soybean actors started producing biodiesel for international market -especially to Europe- and the country quickly became the major biodiesel exporter. In 2010, around twenty processing units started supplying the domestic market. Diesel sold in Argentina contained 5\% of biodiesel. The obligation to add 5\% of biodiesel to diesel has passed to $7 \%$ and the goal is to increase this percentage. The State support and soybean biodiesel competitiveness have been important for biodiesel chain development but some doubts could appear about the kind of actors that could have the benefits.
\end{abstract}

Key words: Argentina, biofuel, soybean biodiesel

réglementés par le gouvernement argentin. Les débouchés à l'exportation sont le fait d'acteurs de moyenne et grande taille, très souvent liés aux activités de trituration.

Sur la base d'un suivi régulier conduit dans le cadre de programmes universitaires de recherche ${ }^{2}$ (entretiens réalisés depuis 2008 auprès d'acteurs-clés producteurs, institutions, chercheurs, compilation d'informations professionnelles et académiques, revue de presse), la réflexion porte sur les raisons et les facteurs de développement de la production d'énergies renouvelables en Argentine, à travers le lancement du

\footnotetext{
2 Programmes "Landuse change, biofuels and rural development in the La Plata Basin (IAI-IRDC, 2008-2010) " et PICT Red "Impactos territoriales de las transformaciones en el sector agroindustrial en la Región Central Argentina. Pasado reciente y escenarios futuros." (Universidad Nacional del Litoral, 2009-2011), "Proyectos de energía en el Noroeste de la Provincia de Buenos Aires: desarrollo territorial e integración regional" (Universidad del Noroeste de la Provincia de Buenos Aires, 2010-2011).
}

biodiesel de soja. Elle est centrée sur I'analyse des stratégies des acteurs, les conditions de production (normes, rentabilité, compétitivité, etc.) et les débouchés. Un premier point concerne les mesures que le gouvernement a prises et qui ont impulsé la production dans le pays. Le deuxième point traite des spécificités de la filière du biodiesel en Argentine, entre abondance de fèves et d'huile brute de soja, valorisation industrielle et atermoiements du soutien public. Le troisième identifie les acteurs impliqués et les marchés concernés ${ }^{3}$.

\section{Le soutien actuel de l'État argentin à la production de biocarburants}

Au début des années 2000, en Argentine, rien ne semblait pouvoir rapprocher monde agricole et monde énergétique, malgré une industrie huilière très

\footnotetext{
${ }^{3}$ Nous remercions chaleureusement $M$. Romain Gaignard pour sa relecture attentive et ses commentaires éclairés.
} 
performante et en constant développement.

L'Argentine avait cependant envisagé cette articulation à la fin des années 1970. En plein choc pétrolier, elle a lancé le Plan "Alconafta » qui préconisait l'utilisation d'alcool de canne à sucre, culture présente dans le nordouest subtropical du pays (à un niveau moindre par rapport au Brésil). Ce plan est abandonné une dizaine d'années plus tard, au profit d'une augmentation croissante de la consommation d'énergies fossiles dont les prix baissaient et les exportations augmentaient.

Or, la structure du transport en Argentine repose sur une consommation considérable de gazole (qui confine au gaspillage énergétique), étant donné le fait que le train a été délaissé et que le tout-routier s'est imposé : les camions de marchandises et les bus de voyageurs sillonnent inlassablement le pays, et avalent les centaines de kilomètres qui séparent les centres urbains et les différentes régions (1 200 kilomètres entre Buenos Aires et Mendoza par exemple). Principal carburant de ces véhicules lourds, le gazole est donc importé en proportions croissantes depuis les années 2000. Pour sa part, le gaz est prisé non seulement pour les usages domestiques et industriels mais aussi pour les véhicules légers (qui roulent également à l'essence). En effet, l'Argentine a le plus grand nombre de voitures à gaz naturel compressé. Leur utilisation par les taxis a été encouragée officiellement dans les années 1980 afin de les substituer à celles qui roulent avec des combustibles pétroliers liquides. Quelques essais avec des biocarburants ont été tentés, mais ils étaient d'envergure limitée et peu valorisés.

L'Argentine aborde par conséquent le $\mathrm{XXI}^{\mathrm{e}}$ siècle avec une dépendance énergétique croissante vis-à-vis des hydrocarbures. $\quad 90 \%$ de l'énergie consommée dans le pays (carburants pour les transports et le secteur agricole, chauffage, etc.) est à base de dérivés de pétrole et de gaz, obtenus en grande partie dans le pays. Les prix au consommateur final sont subventionnés par l'État (intervention récurrente et de plus en plus large depuis le gouvernement de Nestor Kirchner de 2003) en grande partie grâce à la rente pétrolière et aux revenus tirés de la taxation à l'exportation des matières premières comme le soja. Mais des difficultés d'approvisionnement et de distribution énergétique plaident en faveur d'une diversification des sources, en particulier dans les régions éloignées ou en bout de réseaux de distribution. Les distances sont telles que le moindre problème engendre des ruptures d'approvisionnement qui compromettent les activités des personnes et des entreprises.

Ces déficiences énergétiques nationales et régionales ont pour toile de fond le débat international sur l'épuisement des ressources fossiles (Andrich, 2011 ; Dominicis, 2010 ; Cepal, 2009 ; World Bank, 2008). Elles plaident en faveur du remplacement d'une part importante du carburant utilisé par des biocarburants. Le biodiesel et le bioéthanol pourraient être produits de manière abondante, dans un pays excédentaire en matières premières agricoles, exportées sous forme de commodités ou de biens faiblement transformés (cas de I'huile brute de soja et, dans une moindre mesure, du maïs).

Depuis le milieu des années 2000, l'Argentine affiche donc sa volonté de devenir un important producteur de biocarburants, notamment par une politique fiscale incitative (Greenpeace, 2007). En 2001, le gouvernement lance le Plan de competitividad para el combustible Biodiesel (Décret 1396) qui accorde alors des avantages fiscaux. Mais la crise économique de 2001, l'évolution des prix internationaux et nationaux rendent peu viables ou peu compétitifs les projets de production de biocarburants. En 2006, la loi-cadre 26093/2006 définit trois débouchés pour les biocarburants : l'exportation, le marché national (obligatoire à partir de 2010) et l'autoconsommation. Le décret 109/07 permet son application et la production de biodiesel est lancée à grande échelle, d'abord pour l'exportation. La production pour le marché national se concrétise à partir de 2010, lorsque la législation rend obligatoire l'incorporation d'au moins $5 \%$ de biocarburants dans la composition des carburants vendus en Argentine (biodiesel-gazole et éthanol-essence). Le pourcentage a été rapidement élevé à $7 \%$ pour le biodiesel (résolution 554 du Secrétariat à l'Énergie de juillet 2010) (Carrizo et al., 2009; Carrizo, 2010; Droulers et Carrizo, 2011).

En accord avec la loi des biocarburants, quelques mois après son adoption, le Congrès argentin a approuvé la Loi 26.190/2006 pour la production d'électricité à partir d'énergies renouvelables. En mai 2009, l'Assemblée vote le décret d'application (Décret 562) et I'entreprise Enarsa Energía Argentina bras exécutif du ministère de la Planification - lance un appel d'offre à travers le Programme Genren "Provisión de energía eléctrica de fuentes renovables" de 1000 MW. Il est stipulé que $110 \mathrm{MW}$ seront produits à partir de biodiesel. Actuellement, certaines centrales thermiques essayent d'utiliser du biodiesel pour produire de l'électricité ${ }^{4}$.

En janvier 2008, la loi nationale 26.334 octroie des aides aux raffineries de sucre qui commencent, en 2010, à approvisionner le marché national ${ }^{5}$. Neuf raffineries - parmi les vingt-trois en fonctionnement - assurent le volume qui leur a été assigné par le Secrétariat à l'Energie, sur la base d'un mélange à l'essence de $5 \%$ de bioéthanol. Localisées dans les zones sucrières de Tucumán, Salta et Jujuy, à la marge des grands réseaux énergétiques, quelquesunes démarrent aussi la génération complémentaire d'électricité.

Finalement, le cadre réglementaire fondamental est la loi-cadre sur les biocarburants de 2006. Elle préconise la promotion des économies régionales et des petits et moyens producteurs et des PME liées à la filière. Elle annonce des exonérations d'impôts sur les investissements réalisés. Elle prévoit la répartition entre les producteurs des différentes régions et de taille diverse, des volumes de biocarburants nécessaires à l'industrie pétrolière pour procéder au mélange. Enfin, elle réglemente leur prix de vente. Les objectifs sont énergétiques et environnementaux : incorporer de nouvelles sources locales pour la production de carburants et d'électricité, et contribuer à la diminution de l'émission de gaz à effet de serre. Ils sont également économiques et sociaux: alléger la facture énergétique en important moins de

\footnotetext{
${ }^{4}$ Par exemple, I'unité AES à San Nicolas, Province de Buenos Aires, a utilisé du biodiesel pour fournir $5 \%$ du carburant nécessaire à deux machines de $265 \mathrm{MW}$ de puissance (environ $6000 \mathrm{~m}^{3}$ de biodiesel, soit 5300 tonnes approximativement) (Pont, 2011).

${ }^{5}$ Résolution 266/2008 du registre des audits ; résolution 1295/2008 de définition de la qualité ; résolution 689/2009 à propos des entreprises d'éthanol et des volumes à fournir, résolution $733 / 09$, etc.
} 
gazole, élargir la géographie des activités agricoles locales et créer des emplois. Ce dernier aspect relatif au développement des économies régionales (qualifiées de "périphériques " en Argentine) est un argument de fond de la loi. Cependant, il a assez vite rendu perplexe de nombreux acteurs et observateurs. Des emplois ont en effet été créés et des acteurs locaux ont pu se reconvertir vers la production d'énergies renouvelables comme le biodiesel. Mais, les petites structures de type familial n'ont pas été véritablement soutenues et ont été vite confrontées au manque inéluctable de compétitivité face aux projets de grande échelle concentrés dans les terminaux portuaires ou au plus près des zones de consommation les plus peuplées et des grandes villes. Nonobstant, ce cadre législatif et les débouchés qui se profilent sur les deux plans, national et international, ont incité au développement de la capacité productive, d'autant que I'Argentine dispose de quantités importantes d'huile brute de soja ${ }^{6}$.

\section{Sur la base d'une matière première agricole abondante}

En Argentine, I'expansion de la fabrication de biodiesel de soja est au cœur du boum agricole qui s'est emparé, depuis les années 1990, des terres pampéennes et des régions voisines (Villela et al., 2010). Traditionnellement engagés dans les productions de viande bovine, de céréales (blé, maïs) et de tournesol, les producteurs de la Pampa ont très largement adopté la culture du soja. Le protéagineux s'est imposé du fait d'un rapport coût-bénéfice sans égal en ces temps d'innovations biotechnologiques (semences génétiquement modifiées adoptées depuis la campagne 19961997), de méthodes culturales conservatrices (semis direct) et intensifiées (agriculture en continu), de moyens financiers accrus (investisseurs extraagricoles et gestion confiée à des prestataires de service) et d'outils commerciaux et boursiers sophistiqués (Guibert,

\footnotetext{
${ }^{6}$ L'Argentine affiche également ses intentions d'augmenter sa production d'éthanol de canne (quantités faibles) et de débuter celle d'éthanol de maïs, deux biocarburants qui ne sont pas l'objet à proprement parler de cet article.
}

2009 ; Grosso, 2009 ; Guibert et al., 2011). Sa production est passée de 11,4 millions de tonnes en moyenne annuelle durant le quinquennat 19901994, à 16,4 entre 1995 et 1999 . Elle s'envole, ensuite, avec près de 44 millions de tonnes obtenues en moyenne par an entre 2000 et 2004. 49 millions de tonnes ont été récoltées en 2010-2011. L'utilisation de terres pour les productions végétales totales va augmentant : moins de 5 millions d'hectares cultivées en 1990-1991, plus de 10 en 2000-2001, plus de 18 en 2010-2011. Accompagnée par une amélioration des rendements ( $2,8 \mathrm{t} / \mathrm{Ha}$ en moyenne ces dernières années), la culture de soja se fait au détriment des autres cultures et des pâturages (confinement plus fréquent des troupeaux) et par conquête de surfaces dans les régions extrapampéennes (remplacement des cultures vivrières ou locales, et de zones boisées). De fait, les quatre autres cultures-clés souffrent de cette hégémonie du soja. Elles se maintiennent bon an mal an, en ce début des années 2010, à 22 millions de tonnes environ pour le maïs, 15 pour le blé, 2 à 3 pour le sorgho et le tournesol.

La capacité de transformation de l'industrie de trituration localisée en Argentine n'a eu de cesse, à son tour, d'augmenter. Elle atteint, en 2011, environ 160000 tonnes de capacité journalière. Elle est en grande partie concentrée dans quelques unités géantes localisées dans les terminaux portuaires situés le long du Paraná, au Nord et au Sud de Rosario, mais aussi dans des usines importantes, au cœur et au sud-ouest de la Pampa (Informativo semanal de la Bolsa de comercio de Rosario).

Un tiers de I'huile brute de soja obtenue en 2010-2011 (7,1 millions de tonnes) a été consacrée à la production de biodiesel. Cela correspond à un quart (24\%) de la récolte argentine de fèves de soja (12 millions de tonnes) ; autrement dit, la hausse de ces dernières années de la production argentine de soja a été absorbée par la filière biodiesel (Inta, 2011 ; Inta, 2008). En ce qui concerne l'éthanol, son obtention correspond, en 2011, à la canne cultivée sur 325000 hectares. Cette surface ne pouvant pas augmenter considérablement, I'amélioration des rendements est I'enjeu (cf., par exemple, les recherches de la station expérimentale agronomique d'Obispo Colombres, dans la province de Tucumán).
Des producteurs agricoles aux stationsservice, en passant par l'industrie huilière et les entreprises pétrolières (chargées de faire le mélange carburants fossiles-biocarburants), et sans oublier le rôle normatif et incitatif de l'État, toute une chaîne d'acteurs est impliquée dans la production et dans la mise en marché des biocarburants argentins, dont en premier lieu le biodiesel de soja.

\section{Diversité des acteurs, concentration des débouchés}

\section{Des acteurs multiples}

Tandis que les plus grandes usines actuellement habilitées sont la propriété des acteurs de la trituration: Vicentín, Aceitera General Deheza, Molinos Río de la Plata, d'autres, d'une taille petite ou moyenne, ont été pionnières en la matière: Soyenergy, Pitey, Diaser, Biomadero ou AOM.

Selon qu'elles produisent ou pas I'huile brute nécessaire, la CADER (Cámara Argentina de Energías Renovables) distingue trois catégories d'usines de biodiesel (tableau 1, figure 1) :

1) les grandes usines de biodiesel indépendantes car le fait d'acteurs de I'industrie huilière : leur capacité de fabrication est très importante (de 63000 à 480000 tonnes de capacité annuelle installée) et elles disposent de leur propre huile brute ;

2) les autres grandes usines avec une capacité de fabrication également importante (de $50 \quad 000$ à $250 \quad 000$ tonnes) et qui peuvent se procurer I'huile nécessaire assez facilement (proximité géographique ou organisationnelle); et 3) les moyennes et petites usines: leur capacité de fabrication est moindre (en-dessous de 50000 tonnes de capacité installée) et elles ne produisent pas d'huile.

L'ensemble de cette infrastructure a très vite hissé I'Argentine parmi les quatre plus grands producteurs de biodiesel du monde et lui a permis de devenir tout aussi rapidement le premier exportateur (Rozemberg et al., 2009 ; IICA, 2010). Or, des investissements très récents ont augmenté les capacités des unités d'Explora et de Rosario Bio Energy. Trois autres grandes usines ont été homologuées: Cargill (capacité de 


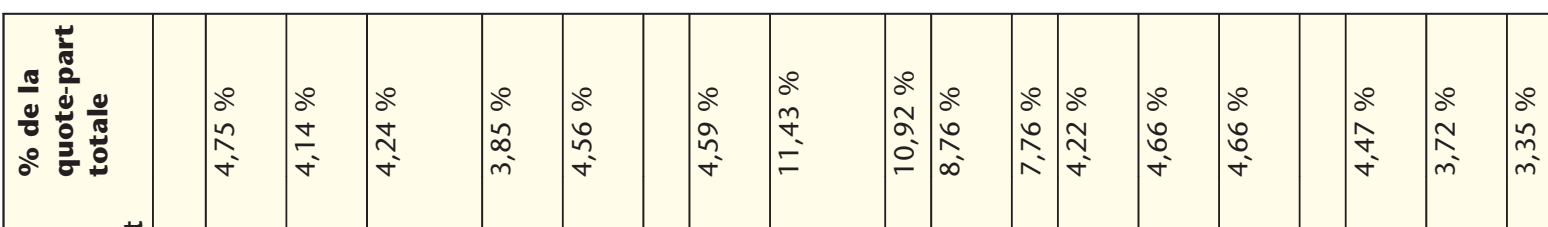

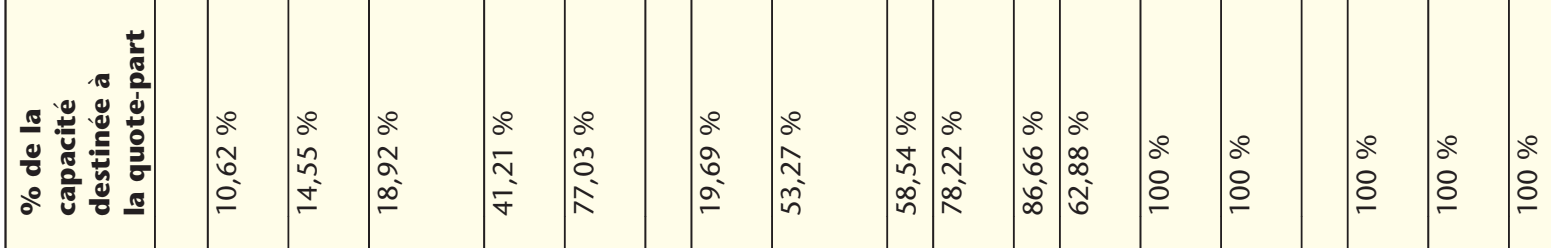

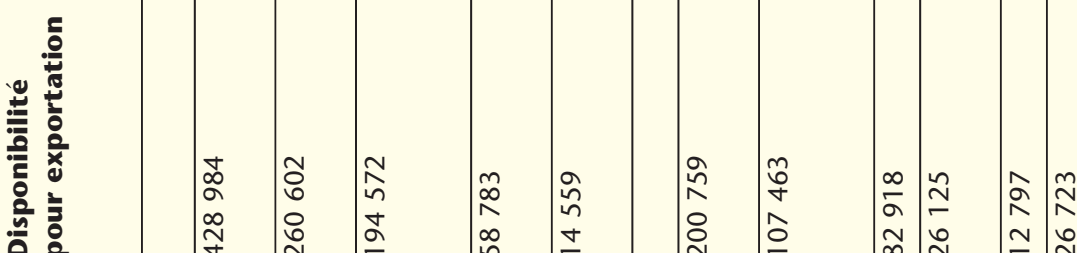

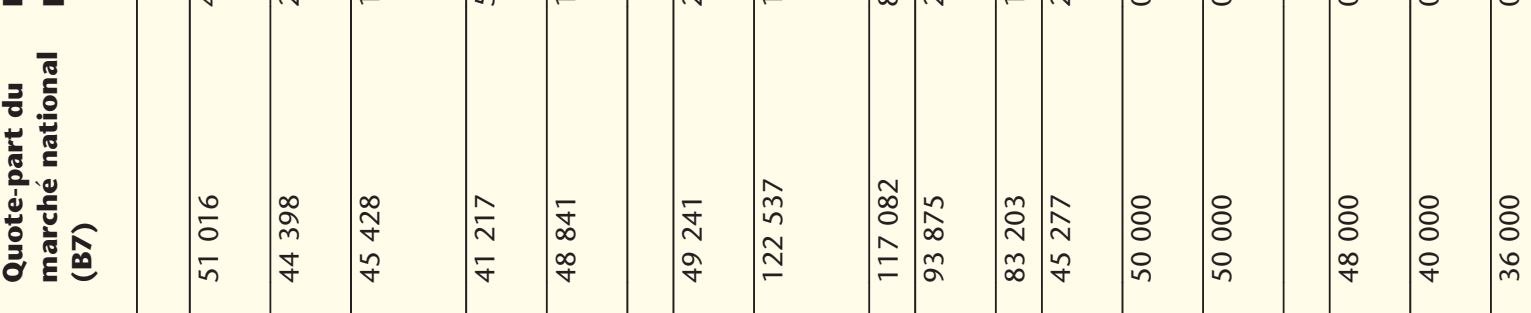

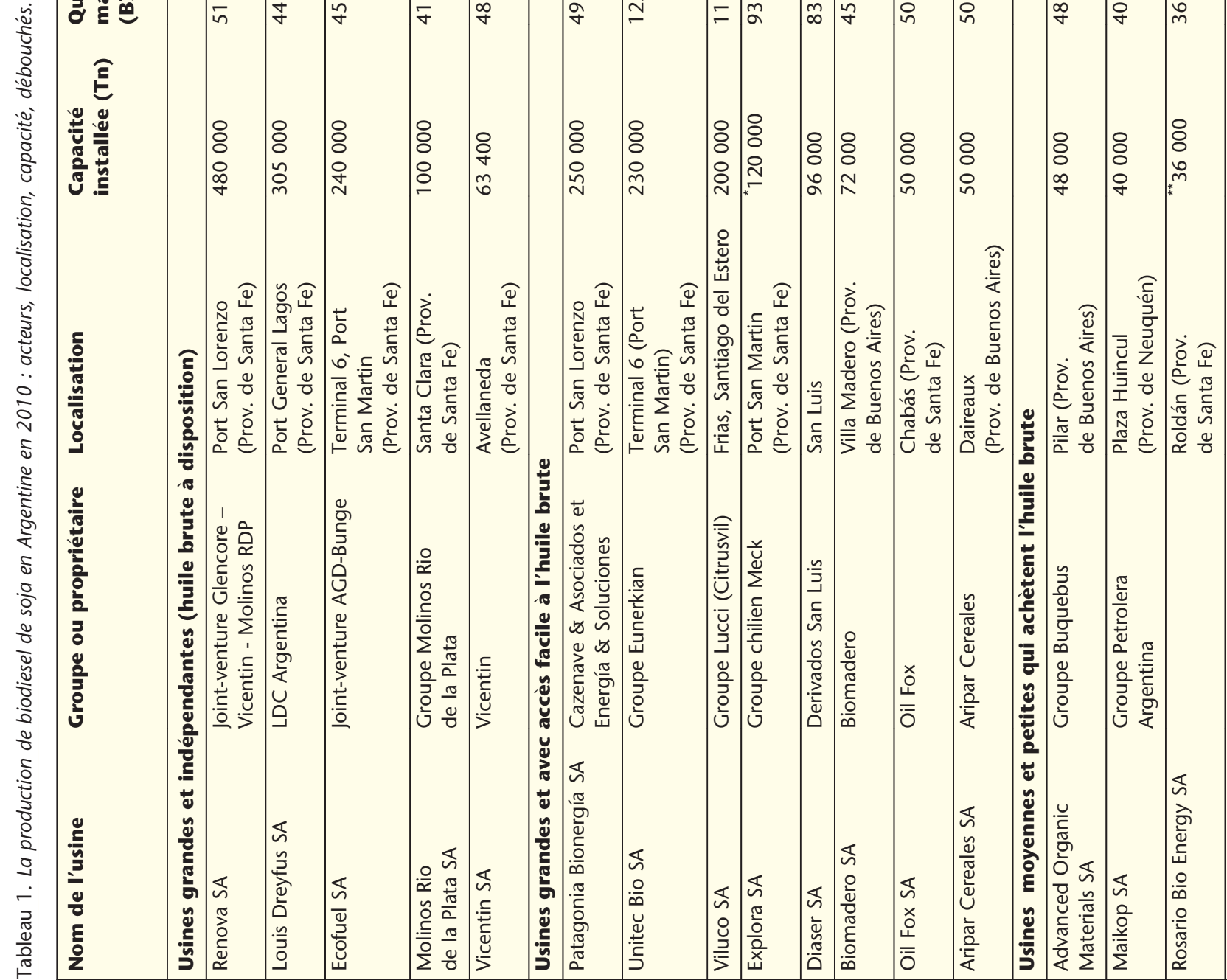




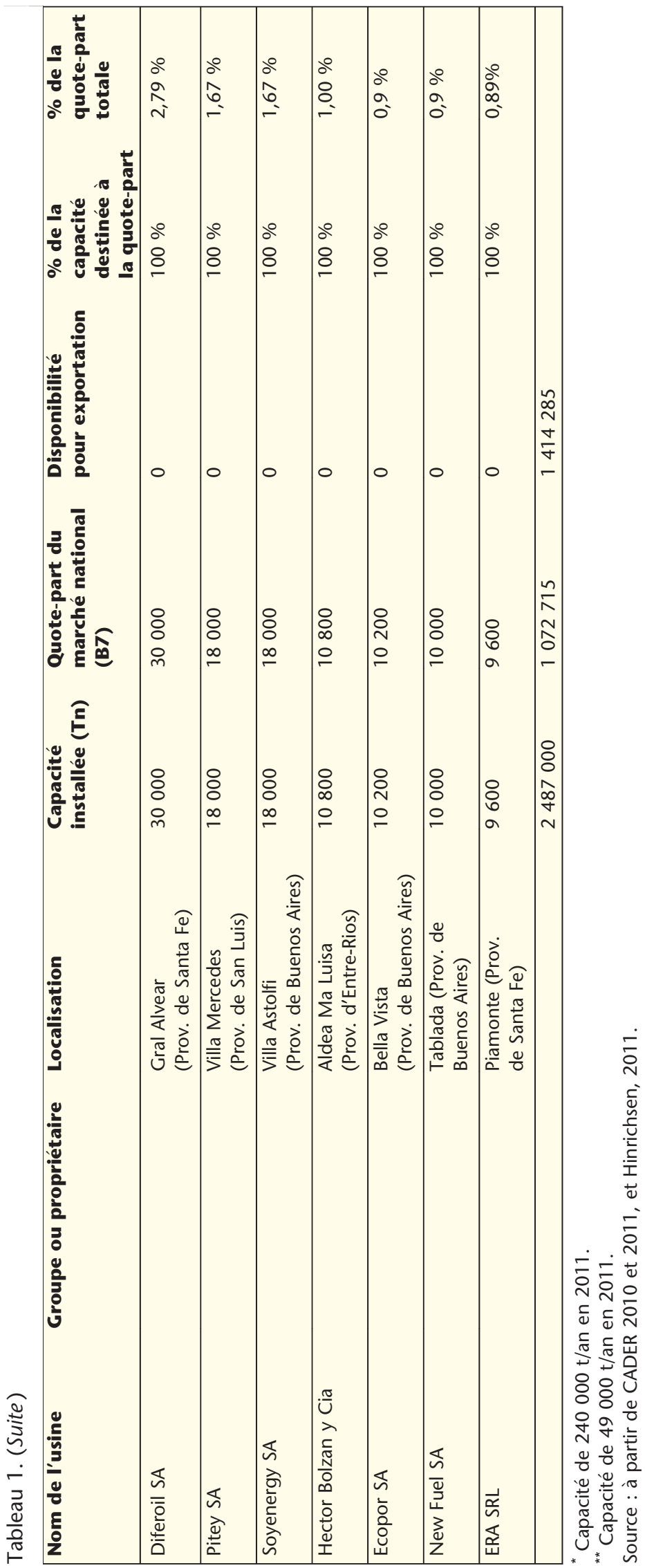

$240000 \mathrm{~T} / \mathrm{an})$; la deuxième usine d'Unitec Bio SA dans le Terminal 6 sur le Paraná (Port de General San Martín dans la Province de Santa Fe) (capacité de 220000 t/an) et BH Biocarburants SRL en Calchaquí (Province de Santa Fe) (capacité de 4000 t/an). En conséquence, la capacité totale installée en Argentine, en 2011, dépasse les 3 millions de t/an (Cader, 2011) et, d'après les projets $d$ 'investissements annoncés, elle devrait passer à 3,5 millions de tonnes par an en 2012 et pourrait dépasser à court terme 5,5 millions de tonnes par an.

\section{Des marchés polarisés}

Jusqu'en 2010, la production de biodiesel de ces usines homologuées était destinée dans sa totalité aux exportations. Celles-ci débutent en 2006 pour 200 millions de dollars ${ }^{7}$ et atteignent 268 millions de dollars en 2007. Les États-Unis et I'Allemagne étaient les deux destinations essentielles, avec respectivement $73 \%$ et $27 \%$ des débouchés. Les taxes sur les exportations, dites " retenciones » et qui ont été réintroduites en 2003 ont un niveau différent selon la commodité considérée. Ainsi, en 2007, elles atteignaient $5 \%$ pour le biodiesel de soja contre plus de $30 \%$ pour l'huile brute, ce qui constitue une incitation du gouvernement à exporter le biocarburant.

En 2007, I'Argentine a exporté plus de 168000 tonnes de biodiesel, soit l'équivalent du total de sa production. En 2008 et 2009, les volumes sont bien plus importants (725 000 t et 1,1 million de t respectivement). En 2010, le lancement du marché national capte environ un quart de la production argentine de biodiesel alors que 1,4 million de tonnes sont exportées (Sanchez, 2011). En 2011, sur 2,4 millions de tonnes de biodiesel produites, 1,5 million ont été exportées. La plus grande partie des exportations prend le chemin de l'Union européenne et le reste est destiné au Pérou. De plus, les exportations ne cessent d'augmenter malgré le fait que les taxes à l'exportation sur le biodiesel soient passées de $5 \%$ en 2007 à $20 \%$ en 2008.

En synthèse, au début du développement de la production de biocarburants en Argentine, en 2007, beaucoup

\footnotetext{
${ }^{7}$ Secrétariat argentin à l'Agriculture.
} 


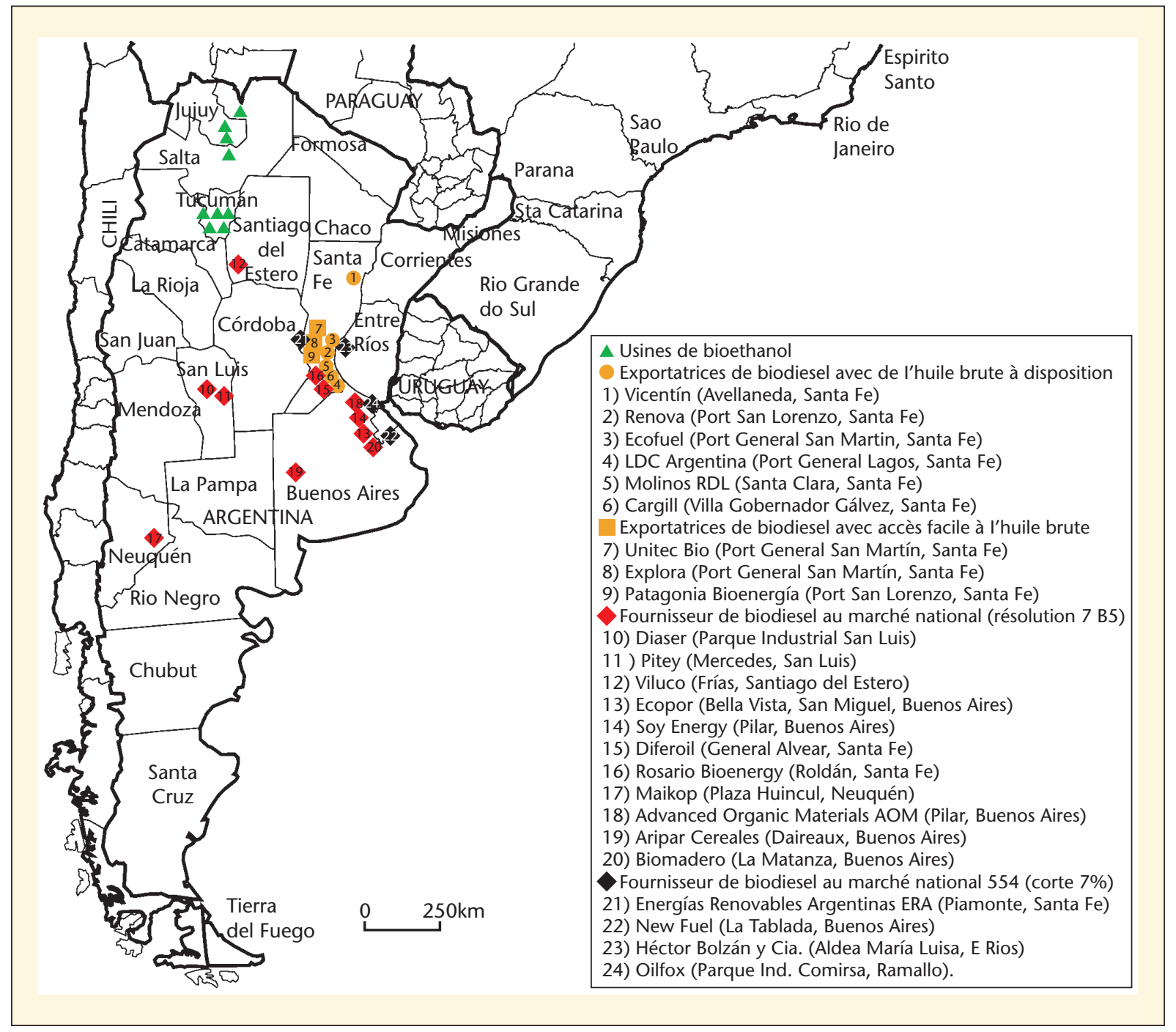

Figure 1. Localisation en Argentine des usines de biodiesel de soja habilitées en 2010.

d'usines de biodiesel étaient en l'état de projet. Les plus petites couvraient, sans respecter parfois certains critères de qualité, l'autoconsommation et une petite demande locale en manque de réglementation. Ce n'est qu'avec la résolution 7/10 de février $2010 \mathrm{du}$ Secrétariat de l'énergie du ministère de la Planification fédérale, des Investissements publics et des Services qu'ont été connus le volume nécessaire pour l'incorporation obligatoire de $5 \%$ de biodiesel (B5) dans le gazole, soit 859819 tonnes pour 2010, les quotas pour chacune des 19 usines (sur la base de leur proposition) et la formule de calcul du prix d'achat par les acteurs pétroliers chargés de réaliser le mélange. En juillet 2010, le secrétariat de l'Énergie a augmenté, à travers la résolution 554/2010, la valeur du mélange à hauteur de $7 \%$ (B7) : 23 usines sont donc habilitées en 2011, afin de fournir aux entreprises pétrolières les 1072715 tonnes nécessaires à ce marché national captif.

Le gouvernement affiche maintenant sa volonté d'atteindre $10 \%$ de biodiesel dans le carburant pour les véhicules (B10). Mais les acteurs pétroliers sont assez réticents : au-delà des changements logistiques et techniques nécessaires, le prix du biodiesel - fixé par le secrétariat de l'Énergie, est plus élevé que celui du gazole (respectivement 5,24 et 4,2 pesos argentins le litre). Du coup, alors que les entreprises se retrouvent avec des excédents de biodiesel, il y a des pénuries récurrentes à la pompe, ce qui oblige à importer du gazole
(Revista " Petroquímica, petróleo, gas \& química ", 2011). La situation est similaire à celle de la France, ce qui révèle le caractère assez ambigu de l'articulation entre des mondes productifs et des enjeux a priori difficilement conciliables (Guindé et al., 2008 ; Jacquet et al., 2007). À ce propos, il convient ici de souligner le type de relations entre les producteurs de biodiesel et les acteurs de la filière pétro-chimique. Les entreprises qui possèdent des raffineries pétrolières $\mathrm{n}$ 'ont pas investi pour obtenir le biocarburant dont l'incorporation est obligatoire. Elles se fournissent en biodiesel auprès des acteurs à qui elles proposent le méthanol nécessaire et avec lesquels elles passent contrat et réalisent le mélange. Ainsi, les quelques projets d'investissement annoncés par 
les compagnies pétrolières présentes en Argentine ne se sont pas concrétisés, ce qui attribue un rôle clair à chaque acteur : le biodiesel de soja est un input proposé aux acteurs des carburants et des énergies par ceux liés au secteur agricole, la relation existant aussi en amont (dans l'autre sens) du fait de l'approvisionnement en méthanol. Le Secrétariat à l'Énergie - en tant qu'autorité d'application de la Loi 20093 - coordonne l'ensemble des activités de la filière, le Ministère de l'économie agissant en tant qu'autorité d'application pour des questions fiscales.

En ce qui concerne la qualité des biocarburants, I'Argentine remplit les normes exigées à l'international. L'importation de technologies de pointe (d'Allemagne ou des États-Unis) et le partenariat Universités/Institut national de la recherche agronomique (INTA)/ acteurs privés conduisent à la mise en œuvre de protocoles de production en accord avec ces normes. La plupart des usines utilisent les dernières technologies (allemandes) et les chercheurs argentins travaillent à la mise au point de process économes en eau et en produits chimiques (méthanol, soude caustique, additifs). Par exemple, un producteur argentin a expérimenté l'obtention de biodiesel en utilisant des ultrasons. De même, certains produisent I'huile brute de soja ou de colza par pression, ce qui permet de s'exempter de l'étape d'extraction du solvant.

\section{Réflexions finales}

Devenue, en quelques années, premier exportateur mondial et quatrième producteur mondial de biodiesel de soja, l'Argentine poursuit depuis 2006 le développement de la production de biocarburants, en stimulant l'augmentation des volumes, la diversification des produits et la multiplication des usages. Les incitations fiscales et la répartition nominative des quotas jouent en faveur de cette progression rapide, respectivement sur les marchés international et national.

Lors de son adoption, la loi-cadre 26093/06 a été présentée comme un outil de développement des économies régionales (hors région de la Pampa), confrontées à une absence de création de valeur ajoutée dans le domaine agricole et fréquemment touchées par la pénurie de produits énergétiques.
Néanmoins, les initiatives qui ont immédiatement surgi ont confirmé la tendance, somme toute évidente, à la concentration spatiale au profit de l'industrie de trituration de grande échelle localisée dans les terminaux portuaires sur le Paraná, ce qui va dans le sens d'une valorisation accrue de ses activités agro-industrielles et agroexportatrices.

Les vicissitudes réglementaires qui ont suivi ont enfin débouché, début 2010, sur la définition des modalités du marché national : répartition des quotas, prix, normes de qualité, pourcentage de mélange. Dans ce climat d'incertitude, les acteurs locaux, de moyenne à petite taille, et attirés par l'approvisionnement du marché national, ont dû attendre. L'attribution des quotas en 2010 donne, également, une place sur ce marché interne aux grandes usines tournées vers l'exportation, ce qui leur permet de bénéficier du prix fixé par le gouvernement et d'atteindre les volumes nécessaires pour le mélange à $7 \%$.

Si les contours législatifs et les choix politiques actuels ont façonné des opportunités commerciales captées par les grands opérateurs localisés dans les terminaux portuaires voués à l'exportation, et si les objectifs de développement régional, qui articulaient production, approvisionnement local et création de valeur ajoutée, semblent bien loin et relèvent de l'illusion, on constate malgré tout l'affirmation d'une filière qui tend à s'étendre spatialement, à multiplier les initiatives commerciales et à dynamiser les innovations scientifiques. La diminution du coût de la facture énergétique et la diversification des sources renforcent l'intérêt national pour appuyer la production de biocarburants.

Le cadre législatif et les débouchés qui ont été déterminés par volonté politique, aussi bien au niveau national qu'international, appuient finalement la création $d^{\prime}$ une filière argentine $d^{\prime}$ une certaine ampleur, sur la base de productions agricoles et agro-industrielles pourvoyeuses des inputs de base (Rastoin et Ghersi, 2010). S'ajoute à la consolidation récente de la filière du biodiesel, l'augmentation des productions d'éthanol de canne (sur la base d'une vingtaine de centrales sucrières) et de mais (cinq projets d'éthanol de maïs viennent d'être approuvés et la mise en fonctionnement de ces usines aurait lieu entre août 2012 et mai 2013).
Néanmoins, il est permis d'être circonspect face à la portée annoncée de ces développements, tant ils sont pris entre incertitudes et ambiguïtés. En Europe, débouché principal actuel de l'Argentine, des restrictions conjoncturelles sont avancées en matière d'importations afin de favoriser les industries nationales (exemple de l'Espagne). Sur le fond, les agro-énergies dites "renouvelables » sont questionnées quant à leur viabilité environnementale (Le Hir, 2011; The Journal of Peasant Studies (2010) et quant à I'acceptation sociale de l'obtention de produits agro-énergétiques, audelà (ou au détriment) de biens alimentaires. Ces incertitudes ne laissent pas indifférents les acteurs qui œuvrent en Argentine, d'autant qu'ils sont déjà ballotés entre des règles nationales actuellement favorables (taxe réduite sur les exportations de biodiesel, mais jusqu'à quand ?) et des revendications transversales (de l'échelle locale à l'échelle mondiale) d'ordre environnemental, économique et social. La mise en cause de la culture en continu, la déforestation, le niveau de rendement énergétique, la rentabilité, sécurité énergétique vs sécurité alimentaire : ces dimensions de la transition énergétique sont essentielles, tout comme celle de la maîtrise des consommations.

\section{RÉFÉRENCES}

Andrich M (comp.). Biocombustibles, energía y alimentos. Buenos Aires : Ed: Antropofagia, 2011.

CADER, Estado de la industria argentina de biochimie. Reporte Cuarto Trimestre 2010: Corte obligatorio de biodiesel ampliado a B7; GENREN y los biocombustibles; ranking mundial de producción. Buenos Aires, 2011. http://www.argentinarenovables.org/ archivos/Estado-Industria-Biodiesel-enero2011. pdf.

CADER (Cámara argentina de energías renovables). Comercio en biodiesel entre Argentina y Europa: propuestas para el desarrollo transparente de una industria. Buenos Aires : 2010. http://www.argentinarenovables.org/informes_ estudios_ensayos.php.

Carrizo S. Nuevos usos en los espacios rurales: la producción de biocombustibles en Argentina. In: Nogar G, Jacinto G. Los espacios rurales: aproximaciones teóricas y procesos de intervención. Buenos Aires: Ed. La Colmena, 2010.

Carrizo S, Guibert M, Berdolini JL. Los actores y mercados de los biocombustibles argentinos: entre incertidumbre y diversificación. EGAL - 
$12^{\circ}$ Encuentro de los geógrafos de América latina, Montevideo, 2009 http://egal2009. easyplanners.info/area06/ (texto $\mathrm{N}^{\circ}$ 6021).

CEPAL. Biocarburants y comercio internacional: una perspectiva latinoamericana. Santiago du Chili : 2009. http://www.eclac.org/publicaciones/xml/1/36181/lcw247e.pdf.

Dominicis A. Les biocarburants. Paris : Ed. Le cavalier bleu, Coll "Idées reçues", 2010.

Droulers M, Carrizo S. Stratégies agro-industrielles autour de la filière des biocarburants au Brésil et en Argentine. Revue Géographique de l'Est 2010 ; 3-4. Puesto en línea el 17 octubre 2011, URL: http://rge.revues.org/ 3107.

Greenpeace. Bionergía: oportunidades y riesgos ¿Qué debe hacer Argentina en materia de biocombustibles? Buenos Aires, 2007. http:// www.greenpeace.org/argentina/es/informes/ bioenerg-a-oportunidades-y-ri/.

Grosso S. "Les pools de culture : diversité des combinaisons financières et productives" p. 223-254. Dossier "Argentine", Revue DÉMÉTER 2010, Paris, Club Déméter, Ed. A. Colin, 2009.

Guibert M, Sili M, Arbeletche P, Piñeiro $D$, Grosso S. Les nouvelles formes d'agricultures entrepreneuriales en Argentine et en Uruguay, Économies et Sociétés, Série "Systèmes agroalimentaires», AG 2011 ; 33 : 1813-1831.

Guibert M. Introduction "L'agriculture argentine au défi de la financiarisation " et "La nouvelle agriculture argentine: entre innovations et incertitudes ". Revue DÉMÉTER $20102009: 143-180$ et 125-141.

Guindé L, Jacquet F, Millet G. Impacts du développement des biocarburants sur la production française de grandes cultures. Revue d'études sur l'agriculture et l'environnement 2008 ; 89 : 55-82.
IICA. Atlas de la agroenergía y los biocombustibles en las Américas: II Biodiésel. Capitulo Argentina. IICA, Programa Hemisférico en Agroenergía y Biocombustibles, San José de Costa Rica, 2010.

Informativo semanal de la Bolsa de comercio de Rosario, différents numéros 2010 et 2011.

INTA (instituto nacional de tecnología agropecuaria). El cambio indirecto del suelo producto del incremento de la producción de biocombustibles. Buenos Aires : $N^{\circ}$ DOC BC-INF-05-11, 2011, http://www.inta.gov.ar/ info/bioenergia/boletines/bc-inf-05-11.pdf.

INTA (instituto nacional de tecnología agropecuaria). Biodiesel production from soybean in Argentina. Buenos Aires: $\mathrm{N}^{\circ}$ DOC IIR-BC-INF-09-08, 2008. http://www. inta.gov.ar/info/bioenergia/boletines/bc-inf09-08.pdf.

Jacquet $F$, Barrière $L$, Bureau JC, Guindé $L$, Millet G, Tréguet D. Les enjeux du développement des biocarburants dans I'Union européenne. Paris: Inra Sciences sociales, $2007 ; 2-3$.

Hinrichsen JJ. SA Corredor-broker. Anuario 2011, 138 p.

Le Hir P. Des biocarburants nocifs pour I'environnement. Le Monde 20/07/2011.

Pochat F. Biocombustibles: un proyecto de sustentabilidad ambiental y económica, Petrotecnia, Buenos Aires, agosto de 2009, 5 p. http://www.petrotecnia.com.ar/agosto09. htm.

Pont S. Los biocombustibles llegan a la generación eléctrica, Revista Nuevas Energías, N5, septiembre-octubre 2011, edición latinoamericana, Buenos Aires.

Rastoin JL, Ghersi G. Le système alimentaire mondial. Concepts et méthodes, analyses et dynamiques. Paris : Ed. Quae, 2010, 565 p.
Revista Petroquímica, petróleo, gas \& química, Con una suba de $36 \%$ en la producción, el biodiesel es la estrella del sector energético, $N^{\circ} 270$, octubre 2011, edición latinoamericana. Buenos Aires.

Rozemberg R, Saslavsky D, Svarzman G. La industria de biocombustibles en Argentina. In : López A, coord. La industria de biocombustibles en el Mercosur. Montevideo : Serie RED Mercosur de investigaciones económicas $\mathrm{N}^{\circ} 15,2009,286$ p.

Sanchez S. Industria argentina de biodiesel : actualidad y perspectivas. Reunión anual de la cadena de valor de biocombustibles. AGD CARBIO, Santa Fe, 2011.

The Journal of Peasant Studies, Politics of biofuels, land and agrarian change, 37, Issue 4 (October 2010). http://www.tandfonline. com/toc/fjps20/37/4.

Villela $\mathrm{F}$, et al. El sistema de agronegocios de la soja en Argentina, su cadena y prospectiva al 2020. Buenos Aires : Universidad de Buenos Aires, 2010.

World Bank. Biofuels: the promise and the risks. 2008. http://go.worldbank.org/ UK40ECPQ20.

\section{POUR EN SAVOIR PLUS}

CADER. Cámara Argentina de Energías Renovables: www.argentinarenovables.org

CARBIO. Cámara Argentina de Biocombustibles : www.carbio.com.ar.

INTA. Instituto Nacional de Tecnología : Agropecuaria www.inta.gov.ar.

Ministerio de Agricultura, ganadería y pesca : www.minagri.gob.ar.

Secretaría de Energía de la República Argentina : www.energia.mecon.gov.ar. 